\title{
THE PROJEGTION OF A LINEAR FUNCTIONAL ON THE MANIFOLD OF INTEGRALS
}

\author{
H. GORDON AND E. R. LORCH
}

\section{ON INTEGRALS}

Preliminaries. If $\mu$ is a measure defined on a space $\mathscr{E}$ and $f_{n}(x)$ is a sequence of $\mu$-integrable functions converging to $f(x)$, then under suitable conditions of boundedness, one has the theorem of Lebesgue that

$$
\lim _{n \rightarrow \infty} \int f_{n}(x) d \mu(x)=\int f(x) d \mu(x) .
$$

In particular,

$$
f_{n}(x) \uparrow f(x) \text { implies } \int f_{n}(x) d \mu(x) \rightarrow \int f(x) d \mu(x) .
$$

Note that the convergence of $\left\{f_{n}(x)\right\}$ to $f(x)$ is pointwise and not uniform. The measure $\mu(x)$ gives rise to a linear functional $F f$ on the space of $\mu$-integrable functions: $F f=\int f(x) d \mu(x)$.

The converse procedure has been studied at length by Daniell. Starting with a suitable collection of functions $f(x)$ where $x$ belongs to an abstract set $\mathscr{E}$, Daniell considers a positive linear functional $F f$ (that is, $f(x) \geqslant 0$ implies $F f \geqslant 0$ ) endowed with the property: $f_{n}(x) \uparrow f(x)$ implies $F f_{n} \rightarrow F f$. He shows that this linear functional has essentially all the properties of an integral - for example, with its help it is possible to extend in the classic fashion the given class of functions $f(x)$ to the class of summable functions. Thus the theorem of Lebesgue and the work of Daniell establish the fact that the existence of an integral is essentially equivalent to the existence of a linear functional with the property:

$$
f_{n}(x) \uparrow f(x) \text { implies } \quad F f_{n} \rightarrow F f .
$$

In the future when we refer to a positive integral, we shall mean a positive linear functional which has the property (2). When we refer to an arbitrary integral we shall mean a linear functional $F$ which is the difference of two positive integrals: $F=F_{1}-F_{2}, F_{1} \geqslant 0, F_{2} \geqslant 0$.

The aim of this note is to establish a decomposition theorem for linear functionals on particular Banach spaces of functions. Thus given an abstract set $\mathscr{E}$ and a Banach space $\mathfrak{B}$ of functions $f(x), x \in \mathscr{E}$, which will be described more fully below, let $F$ represent a bounded linear functional on $\mathfrak{B}$. We shall see that there is a unique decomposition $F=G+H$ where $G$ is an integral and $H$ is purely finitely additive in a sense to be defined. Furthermore, if $F$

Received December 1, 1955. This work was carried out with the help of a grant from the National Science Foundation. The result contained in this paper was reported in (1). 
is positive, $F \geqslant 0$, then also $G \geqslant 0$ and $H \geqslant 0$. If $F \geqslant 0$ and $H \geqslant G$, then $G=0$.

This theorem may be considered to be an abstract formulation of a result in the theory of measure which has been noted by several authors ${ }^{1}$ and which has received its most complete formulation from Yosida and Hewitt (3). The latter consider an additive positive measure $\phi$ defined on a set $X$ and prove that there exist positive measures $\phi_{c}, \phi_{p}$ such that $\phi_{c}$ is completely additive and $\phi_{p}$ is purely finitely additive and such that $\phi=\phi_{c}+\phi_{p}$.

The well-known Riesz representation theorem states that every linear functional on the space $C$ of real continuous functions $f(x), 0 \leqslant x \leqslant 1$, may be represented as an integral of the form

$$
\int_{0}^{1} f(x) d \alpha(x)
$$

This theorem has been generalized to the following form. Let $\mathscr{R}$ be a compact Hausdorff space and let $C(\mathscr{R})$ be the Banach space of continuous functions $f(x)$ with the norm $\|f\|=1$.u.b. $|f(x)|, x \in \mathscr{R}$. Then if $F$ is a linear functional on $C(\mathscr{R})$, there is a completely additive measure $\mu(x)$ defined over the Borel sets of $\mathscr{R}$ such that

$$
F f=\int_{\mathscr{R}} f(x) d \mu(x) .
$$

In other words, every linear functional is an integral in our sense. However, the latter result is immediate. For if $\left\{f_{n}(x)\right\}$ is a monotone sequence of functions converging to $f(x)$, then since $\mathscr{R}$ is compact, pointwise convergence implies uniform convergence. Thus $f_{n}(x) \uparrow f(x)$ implies $\left\|f-f_{n}\right\| \rightarrow 0$ which in turn implies $F f_{n} \rightarrow F f$ for an arbitrary bounded linear $F$.

Example. We give an example of a linear functional $F$ which does not have property (2) and hence is not an integral.

Let $f(x)$ be a continuous function defined on the real line which vanishes outside a compact set. Define the functional $F$ by $F f=0$. The totality of such functions constitutes a linear manifold. Consider the closure $\mathfrak{M}$ of this manifold in the uniform topology; on $\mathfrak{M}$ let $F$ be defined by continuity (to be zero). We adjoin to $\mathfrak{M}$ the set of constants $\lambda$ and define $F \lambda=\lambda$. Then the system so obtained is a Banach space and $F$ is a bounded linear functional on this space. There exists a sequence $\left\{f_{n}(x)\right\}$ of functions each of which vanish outside a compact set and such that $f_{n}(x) \uparrow 1$ for all $x$. Evidently $0=F f_{n}(x) \neq F 1=1$.

\section{The Fundamental Projection}

The Space. Let $\mathscr{E}$ be an abstract set. Consider a set $\mathfrak{B}$ of bounded real functions on $\mathscr{E}$. Suppose that $\mathfrak{B}$ has the properties:

\footnotetext{
${ }^{1}$ Woodbury (2) mentions that the result for measures was known to B. Jessen. See also the paper of Yosida and Hewitt (3, footnote 2), whose decomposition theorem was also known to Kakutani. (Added in Proof: See also recent work of H. Bauer, in particular, Math. Z., 65 (1956), 448-482.)
} 
(1) $\mathfrak{B}$ is a vector space - that is, $f(x), g(x) \in \mathfrak{B}$ imply $\alpha f(x)+\beta g(x) \in \mathfrak{B}$ for real $\alpha, \beta$;

(2) $\mathfrak{B}$ is a lattice - that is, $f(x)$ and $g(x) \in \mathfrak{B}$ imply that $f(x) \wedge g(x)=\inf$ $(f(x), g(x))$ and $f(x) \vee g(x)=\sup (f(x), g(x)) \in \mathfrak{B}$;

(3) $\mathfrak{B}$ is closed in the uniform topology-that is, $\mathfrak{B}$ contains the uniform limit of any sequence in $\mathfrak{B}$. If we write

$$
\|f\|=1 . \text { u.b. }|f(x)|, x \in \mathscr{E}
$$

then $\mathfrak{B}$ is a real Banach space. It may be noted that if $\mathfrak{B}$ is an algebra, condition (2) is dependent on the remaining hypotheses.

We shall be interested in the space $\mathfrak{B}^{*}$ consisting of all bounded linear functionals defined on $\mathfrak{B}$. If the functional $F \in \mathfrak{B}^{*}$ has the property $F f \geqslant 0$ for each $f(x) \geqslant 0$ then $F$ is called positive.

The following theorem is proved by methods well known in the theory of measure.

If $F$ is an arbitrary bounded linear functional over $\mathfrak{B}$, there exist two positive functionals $G$ and $H$ such that $F=G-H$. Furthermore $G$ and $H$ are bounded and satisfy $\|G\| \leqslant\|F\|,\|H\| \leqslant\|F\|$.

Proof. If $f(x) \in \mathfrak{B}$, then there exist positive functions $g(x), h(x) \in \mathfrak{B}$ such that $f(x)=g(x)-h(x)$. Indeed, we may take $g(x)=f(x) \vee 0$ and $h(x)$ $=(-f(x)) \vee 0$. Since $F f=F(g-h)=F g-F h, F$ is completely known if it is known on the cone of positive elements.

Let $f(x) \geqslant 0$ be fixed and define

$$
G f=\underset{0 \leqslant h \leqslant f}{1 . u . b .} F h .
$$

Then for positive $f, f_{1}$, and $f_{2}$ it may be seen that $G\left(f_{1}+f_{2}\right)=G f_{1}+G f_{2}$ and if $\alpha>0, G \alpha f=\alpha G f$. It is necessary to use the lattice properties of $\mathfrak{B}$ in order to establish this fact. Thus, if for positive functions $f_{i}, g_{i}$ we have $f_{1}+g_{1}$ $=f_{2}+g_{2}$, then $G f_{1}-G f_{2}=G g_{2}-G g_{1}$. The definition of $G$ is completed as follows: If $f$ is arbitrary, let $f=g-h$ where $g$ and $h$ are positive, and set $G f=G g-G h$. It may be seen that $G$ is a linear functional. If $f \geqslant 0$, since

$$
G f=\underset{0 \leqslant h \leqslant f}{1 . u . b .} \quad F h,
$$

$G f \geqslant 0$; that is, $G$ is positive. Finally $G$ is bounded and for the bounds of $G$ and $F$ we have $\|G\| \leqslant\|F\|$. This follows from the inequalities

$$
G f=G\left(f^{+}-f^{-}\right)=G f^{+}-G f^{-} \leqslant G f^{+} \leqslant\|F\|\|f+\| \leqslant\|F\|\|f\| .
$$

Similarly $G f \geqslant-\|F\|\|f\|$. Here $f^{+}$and $f-$ denote the positive and negative parts of $f$. Thus $\|G\| \leqslant\|F\|$.

The linear functional $H$ is defined by $H=G-F$ or by

It is clear that $\|H\| \leqslant\|F\|$.

$$
H f=\underset{0 \leqslant g \leqslant f}{\operatorname{lu}_{0} . \mathrm{b} .}(-F g) .
$$


We may now prove that the totality of integrals over $\mathfrak{B}$ is a closed linear manifold. If $F_{1}$ and $F_{2}$ are integrals (that is, each is the difference of two positive integrals), then obviously $c_{1} F_{1}+c_{2} F_{2}$ is also an integral. Thus the integrals form a linear manifold. We shall see that this manifold is closed.

Let $\left\{F_{m}\right\}$ be a sequence of integrals and let $F$ be a linear functional such that $\left\|F_{m}-F\right\| \rightarrow 0$. Suppose $F_{m}=G_{m}-H_{m}$ where $G_{m}$ and $H_{m}$ are positive integrals. Suppose $\left\{f_{n}(x)\right\}$ is a sequence of functions converging in a pointwise monotone manner to $0, f_{n}(x) \downarrow 0$.

Since (by the previous theorem) we may write $F=F^{+}-F^{-}$, we have by the definition of $F^{+}$: There exists a function $g_{n}(x)$ such that $0 \leqslant g_{n}(x) \leqslant f_{n}(x)$, and $F^{+} f_{n} \leqslant F g_{n}+2^{-n}$. Thus, since $F=F-F_{m}+G_{m}-H_{m}$,

or

$$
F^{+} f_{n} \leqslant\left(F-F_{m}\right) g_{n}+\left(G_{m}-H_{m}\right) g_{n}+2^{-n}
$$

$$
F^{+} f_{n} \leqslant\left\|F-F_{m}\right\|\left\|f_{1}\right\|+G_{m} f_{n}+H_{m} f_{n}+2^{-n} .
$$

To show that $F^{+} f_{n} \rightarrow 0$, note that by choosing $m$ large but fixed, $\left\|F-F_{m}\right\|$ $\left\|f_{1}\right\|$ can be made arbitrarily small, and then, since $G_{m}$ and $H_{m}$ are positive integrals, the remaining terms on the right may be made small at will by letting $n \rightarrow \infty$. Thus $F^{+}$is an integral. Similarly $F^{-}$is an integral and hence finally $F=F^{+}-F^{-}$is also an integral. This proves that the integrals form a closed linear manifold.

The Projection Operator. We shall now introduce a bounded linear transformation $T$ whose domain and range is the space $\mathfrak{B}^{*}$ of linear functionals. $T$ will be defined first for positive linear functionals.

Let $F \in \mathfrak{B}^{*}$ be a fixed positive functional. Consider an arbitrary positive $f \in \mathfrak{B}$. Let $\left\{f_{n}(x)\right\}$ be an increasing sequence of positive functions in $\mathfrak{B}$ converging pointwise to $f, f_{n}(x) \uparrow f(x)$. Then $\left\{F f_{n}\right\}$ is an increasing sequence and since $F f_{n} \leqslant F f$, the sequence $\left\{F f_{n}\right\}$ has a limit $\geqslant 0$. Now consider the class $\mathscr{K}$ of all sequences $\left\{f_{n}\right\}$ such that $f_{n} \uparrow f$ and the class of all limits of the sequences $\left\{F f_{n}\right\}$. The greatest lower bound of these sequences is a number which depends on $F$ and $f$ and which we denote by $G f$. Thus we may write

We first establish

$$
G f=\text { g.l.b. }\left(\lim _{u} F f_{n}\right), \quad\left\{f_{n}\right\} \in \mathscr{K} .
$$

Lemma 1. The functional $G$ is linear and positively homogeneous on the positive functions in $\mathfrak{B}$. That is, if $f(x) \geqslant 0, g(x) \geqslant 0$ and $\alpha \geqslant 0$ are given, then $G(f+g)=G f+G g$ and $G(\alpha f)=\alpha G f$.

Proof. Let $f(x) \geqslant 0$ and $g(x) \geqslant 0$ be given. For a given $\epsilon>0$ let $\left\{f_{n}(x)\right\}$ be a sequence such that $f_{n}(x) \uparrow f(x)$ and $\lim F f_{n} \leqslant G f+\epsilon$. Similarly let $\left\{g_{n}(x)\right\}$ be a sequence such that $g_{n}(x) \uparrow g(x)$ and $\lim F g_{n} \leqslant G g+\epsilon$. Then $\left\{f_{n}(x)+g_{n}(x)\right\}$ converges to $f(x)+g(x)$ and hence

$$
G(f+g) \leqslant \lim F\left(f_{n}+g_{n}\right)=\lim F f_{n}+\lim F g_{n} \leqslant G f+G g+2 \epsilon .
$$

This argument implies that $G(f+g) \leqslant G f+G g$. 
Now suppose that $\left\{h_{n}(x)\right\}$ is a sequence such that $h_{n}(x) \uparrow f(x)+g(x)$ and $G(f+g) \geqslant \lim F h_{n}-\epsilon$. Let $f_{n}(x)=f(x) \wedge h_{n}(x)$ and write $g_{n}=h_{n}-f_{n}$. Then $f_{n}(x) \uparrow f(x)$ and $g_{n}(x) \uparrow g(x)$. Thus

$$
G(f+g)+\epsilon \geqslant \lim F h_{n}=\lim F f_{n}+\lim F g_{n} \geqslant G f+G g .
$$

This means that $G(f+g) \geqslant G f+G g$. Joining this inequality to the previous one, we have $G(f+g)=G f+G g$. Obviously

$$
G \alpha f=\alpha(G f), \quad \alpha \geqslant 0, \quad f \geqslant 0 .
$$

The functional $G$ is now extended to the whole of the space $\mathfrak{B}$ in the following manner. We have noted that if $f \in \mathfrak{B}$, then $f$ may be expressed as the difference of two positive functions; for example $f=f+-f-$. Now let $f$ be arbitrary in $\mathfrak{B}$ and suppose $f=g-h$ where $g \geqslant 0$ and $h \geqslant 0$. Define $G f=G g-G h$. The definition is valid, for if $g-h=g^{\prime}-h^{\prime}$,

$$
G g+G h^{\prime}=G\left(g+h^{\prime}\right)=G\left(g^{\prime}+h\right)=G g^{\prime}+G h .
$$

Lemma 2. The functional $G$ is bounded, positive, and linear over $\mathfrak{B}$. Furthermore

$$
\|G\| \leqslant\|F\|
$$

Proof. If $f_{1}, f_{2} \in \mathfrak{B}$ write $f_{1}=g_{1}-h_{1}, f_{2}=g_{2}-h_{2}$ where $g_{i}, h_{i}$ are positive. Then

$$
\begin{array}{r}
G\left(f_{1}+f_{2}\right)=G\left(\left[g_{1}+g_{2}\right]-\left[h_{1}+h_{2}\right]\right)=G\left(g_{1}+g_{2}\right)-G\left(h_{1}+h_{2}\right) \\
=G g_{1}+G g_{2}-G h_{1}-G h_{2}=G f_{1}+G f_{2} .
\end{array}
$$

If $\alpha \geqslant 0$, then

$$
G \alpha f=G(\alpha[g-h])=G(\alpha g-\alpha h)=G \alpha g-G \alpha h=\alpha[G g-G h]=\alpha G f .
$$

Similarly if $\alpha \leqslant 0$.

In proving that $G$ is bounded, we keep in mind that $F$ is a positive functional. From the definition (3) it is obvious that $G$ is also positive. Thus for $f=f+-f-$ we have

$$
G f=G f^{+}-G f^{-} \leqslant G f^{+} \leqslant F f^{+} \leqslant\|F\|\|f+\| \leqslant\|F\|\|f\| .
$$

Similarly $G f \geqslant-\|F\|\|f\|$. Hence $G$ is bounded and $\|G\| \leqslant\|F\|$.

Thus we have established a mapping $F \rightarrow G$ of the set of bounded positive linear functionals into itself. We shall write $T F=G$. The mapping function $T$ has the properties indicated in

Lemma 3. If $F, F_{1}$ and $F_{2}$ are bounded positive linear functionals and $\alpha \geqslant 0$, then $T\left(F_{1}+F_{2}\right)=T F_{1}+T F_{2}$ and $T(\alpha F)=\alpha T F$.

Proof. Let $F_{1} \geqslant 0, F_{2} \geqslant 0$ and let $f \geqslant 0$. For a given $\epsilon>0$, let $\left\{f_{n}\right\}$ be such that $f_{n} \uparrow f$ and

$$
\lim \left(F_{1}+F_{2}\right) f_{n} \leqslant T\left(F_{1}+F_{2}\right) f+\epsilon .
$$


Then

$$
\begin{aligned}
T\left(F_{1}+F_{2}\right) f+\epsilon \geqslant \lim \left(F_{1}+F_{2}\right) f_{n}=\lim F_{1} f_{n}+\lim F_{2} f_{n} \\
\geqslant T F_{1} f+T F_{2} f .
\end{aligned}
$$

Now let $\left\{f_{i n}\right\}, i=1,2$, be so chosen that $f_{i n} \uparrow f, T F_{i} f+\epsilon \geqslant \lim F_{i} f_{i n}$. Let $f_{n}=f_{1 n} \wedge f_{2 n}$. Then $f_{n} \uparrow f$ and $\lim F_{i} f_{n} \leqslant \lim F_{i} f_{\text {in }}$. Hence

$$
\begin{aligned}
T F_{1} f+T F_{2} f+2 \epsilon & \geqslant \lim F_{1} f_{1 n}+\lim F_{2} f_{2 n} \geqslant \lim F_{1} f_{n} \\
& +\lim F_{2} f_{n}=\lim \left(F_{1}+F_{2}\right) f_{n} \geqslant T\left(F_{1}+F_{2}\right) f .
\end{aligned}
$$

This and the inequality of the preceding paragraphs prove that $T\left(F_{1}+F_{2}\right) f$ $=T F_{1} f+T F_{2} f$. This fact has been established for $f \geqslant 0$. It obviously holds for arbitrary $f$. Thus $T\left(F_{1}+F_{2}\right)=T F_{1}+T F_{2}$. The proof that $T \alpha F=\alpha T F$ runs along similar lines.

We now extend $T$ to all of $\mathfrak{B}^{*}$. If $F$ is arbitrary in $\mathfrak{B}^{*}$, then there exist positive functionals $G$ and $H$ such that $F=G-H$. We define: $T F=T G-T H$. By Lemma 3, the definition is admissible.

LEMMA 4. The transformation $T$ defined above is a bounded and linear transformation of $\mathfrak{B}^{*}$ into itself. Furthermore if $F$ is a positive integral, $T F=F$.

Proof. The proof of linearity is straightforward.

We have seen that if $F$ is positive, $T F$ is positive and hence

$$
T F f=T F\left(f^{+}-f^{-}\right) \leqslant T F f+\leqslant F f+\leqslant\|F\|\|f\| .
$$

Similarly $T F f \geqslant-\|F\|\|f\|$. Hence $\|T F\| \leqslant\|F\|$. Now let $F$ be arbitrary and write $F=F^{+}-F^{-}$where $F^{+}$and $F^{-}$are the positive and negative parts of $F$. Then

$$
\|T F\| \leqslant\left\|T F^{+}\right\|+\left\|T F^{-}\right\| \leqslant\left\|F^{+}\right\|+\left\|F^{-}\right\| \leqslant 2\|F\|
$$

by the immediately preceding argument.

Finally, if $F$ is a positive integral, then by definition, for any $f \geqslant 0$ and positive sequence $\left\{f_{n}\right\}$ with $f_{n} \uparrow f$ we have by $(2), F f_{n} \rightarrow F f$ hence $T F f=F f$. If $f$ is arbitrary, the same equation holds, hence $T F=F$.

Lemma 5. The transformation $T$ is a projection, that is, $T^{2}=T$. The range of the projection consists precisely of all integrals in $\mathfrak{B}^{*}$.

Proof. The definition of $G=T F$ for positive $F$ was given in terms of sequences of functions $\left\{f_{n}(x)\right\}$ which converge upward to $f(x)$-see (3). However we may also use series of functions. In fact, let $f(x) \geqslant 0$ be arbitrary and let $\left\{g_{n}(x)\right\}$ be a sequence of positive functions such that $\Sigma g_{n}(x)=f(x)$. Let $\mathscr{L}$ be the class of all such sequences. Then clearly, we may write (3) in the alternative form

$$
G f=\text { g.l.b. }\left(\sum_{n} F g_{n}\right), \quad\left\{g_{n}\right\} \in \mathscr{L} .
$$


Now, let $f(x) \geqslant 0$ and let $F \geqslant 0$. Let $f=\sum f_{n}$ where $\left\{f_{n}\right\}$ is any sequence of positive functions. Let $\epsilon>0$ be given. We find positive functions $g_{n m}$ such that

$$
f_{n}=\sum_{m} g_{n m}, \quad T F f_{n} \geqslant \sum_{m} F g_{n m}-2^{-n} \epsilon, \quad n=1,2, \ldots
$$

Since $T F \geqslant 0, \sum_{n} T F f_{n} \leqslant T F f$ and thus $\sum_{n} \sum_{m} F g_{n m}<\infty$.

More precisely

$$
\sum_{n} T F f_{n} \geqslant \sum_{n, m} F g_{n m}-\epsilon
$$

Since

$$
\sum_{n, m} g_{n m}=f, \quad T F f \leqslant \sum_{n, m} F g_{n m} .
$$

Substituting in (4) we have $T F f \leqslant \sum T F f_{n}+\epsilon$. Since $\epsilon$ is arbitrary, this gives $T F f \leqslant \sum T F f_{n}$. Since, obviously, $\sum T F f_{n} \leqslant T F f$, we have $\sum T F f_{n}=T F f$. Now according to $\left(3^{\prime}\right)$

$$
T^{2} F f=\text { g.l.b. } \sum_{n} T F f_{n}, \quad\left\{f_{n}\right\} \in \mathscr{L} ;
$$

thus $T^{2} F f=T F f$. The latter identity holds for any $f \geqslant 0$ and $F \geqslant 0$ and this leads to the conclusion $T^{2}=T$.

To finish the proof of the lemma, it is necessary to show that the range of $T$ is precisely the manifold of integrals. If $F$ is an integral, then by definition $T F=F$. Next suppose that $F \geqslant 0$ and that $T F=F$. This is precisely the statement that $F$ is an integral. Suppose now that $F$ is arbitrary and that $T F=F$. Writing $F=F^{+}-F^{-}$we have

$$
F=T F=T F^{+}-T F^{-}
$$

and since $T^{2}=T$,

$$
T^{2} F^{+}=T F^{+}, \quad T^{2} F^{-}=T F^{-} .
$$

Now $T F^{+} \geqslant 0$ and $T F^{-} \geqslant 0$ and hence both are integrals. Finally, $F$, which is the difference of two integrals, is an integral. This completes the proof of the lemma.

Lemma 6. The transformations $T$ and $I-T$ are positive: that is, $F \geqslant 0$ implies $T F \geqslant 0$ and $(I-T) F \geqslant 0$.

From the definition of $T$, it is obvious that $F \geqslant 0$ and $f \geqslant 0$ imply $T F f \geqslant 0$ and $F f \geqslant T F f$. This is equivalent to the statement of the lemma.

We now obtain a characterization of the functionals $H$ such that $T H=0$.

Lemma 7. Let $H \geqslant 0$ and $T H=0$. Suppose $G \geqslant 0$ is an integral $(T G=G)$ and that $H \geqslant G$. Then $G=0$.

We have $H \geqslant G \geqslant 0$. Since $T$ is positive, $0=T H \geqslant T G=G \geqslant 0$, hence $G=0$.

Lemma 8. Let $H \geqslant 0$ have the property that whenever $G \geqslant 0$ is such that $T G=G$ and $H \geqslant G$, then $G=0$. Then $T H=0$. 
Since $I-T$ is positive, $(I-T) H \geqslant 0$, that is $H \geqslant T H$. However $T H \geqslant 0$ and $T(T H)=T H$. Thus by the hypothesis concerning $G, T H=0$.

Lemma 9. The projection $T$ is uniquely defined by the properties given in lemmata $5,6,7,8$.

Let $T_{i}(i=1,2)$ be two projections having the indicated properties. Let $\mathfrak{M}_{i}$ be the set of $G$ such that $T_{i} G=G$. Similarly let $\mathfrak{N}_{i}$ be the set of $H$ such that $T_{i} H=0$. To prove the lemma, it will be sufficient to show that $\mathfrak{M}_{1}=\mathfrak{M}_{2}$ and $\mathfrak{R}_{1}=\mathfrak{R}_{2}$. The equality $\mathfrak{M}_{1}=\mathfrak{M}_{2}$ is given in Lemma 5 .

Before proving that $\mathfrak{R}_{1}=\mathfrak{R}_{2}$ we note that $\mathfrak{R}_{i}$ is determined by its positive elements. For let $F$ be arbitrary and write $F=F^{+}-F^{-}$. Then the general element in $\mathfrak{R}_{i}$ is

$$
\left(I-T_{i}\right) F=\left(I-T_{i}\right) F^{+}-\left(I-T_{i}\right) F^{-} .
$$

Both of these functionals are positive since $I-T_{i}$ is positive.

Now let $H \geqslant 0$ be in $\Re_{1}$. Then $T_{1} H=0$ and hence if $G$ is a positive integral such that $H \geqslant G$, then $G=0$ by Lemma 7 . Thus by Lemma $8, T_{2} H=0$, that is $H \in \mathfrak{N}_{2}$. This shows that $\mathfrak{N}_{1} \subset \mathfrak{N}_{2}$. Since the argument is reversible, $\mathfrak{N}_{1}=\mathfrak{N}_{2}$.

We shall recapitulate these results into our fundamental

THEOREM A. In the space of linear functionals over the real space $\mathfrak{B}$ there is one and only one projection $T$ with the properties:

(a) $T G=G$ if and only if $G$ is an integral.

(b) The transformations $T$ and $I-T$ are positive.

\section{iII. Complex Spaces}

Up to the present, we have considered real spaces only. We turn to a brief discussion of some details which will show that the theory applies to complex spaces as well. Consider as before a real vector space $\mathfrak{B}_{r}$ consisting of certain bounded real valued functions defined on an abstract set $\mathscr{E}$. $\mathfrak{B}_{r}$ is assumed to be algebraically closed under the lattice operations $f \vee g$ and $f \wedge g$ and topologically closed with respect to uniform convergence. Consider now the set $\mathfrak{B}_{c}$ of all complex-valued functions $f(x)=f_{1}(x)+i f_{2}(x)$ where $f_{1}(x)$, $f_{2}(x) \in \mathfrak{B}_{r}$. If we set

$$
\|f\|=\left(\left\|f_{1}\right\|^{2}+\left\|f_{2}\right\|^{2}\right)^{\frac{1}{2}},
$$

it is clear that $\mathfrak{B}_{c}$ is a complex Banach space. Also $\mathfrak{B}_{r}$ is a subset of $\mathfrak{B}_{c}$.

If $F$ is a bounded linear functional on $\mathfrak{B}_{r}$, it may be extended in a natural way to $\mathfrak{B}_{c}$. This is done by defining

$$
F f(x)=F\left(f_{1}(x)+i f_{2}(x)\right)=F f_{1}(x)+i F f_{2}(x) .
$$

The bound of the extended functional $F$ is the same as that of the restricted 
$F$. Functionals $F$ and $G$ of this type may be added and multiplied by complex scalars as follows:

$$
(F+G) f=F f+G f, \quad[(\alpha+i \beta) F] f=\alpha F f+i \beta F f .
$$

Thus they constitute a linear manifold contained in $\mathfrak{B}_{c}{ }^{*}$. We shall show that this linear manifold coincides with $\mathfrak{B}_{c}{ }^{*}$.

To this effect, let $F$ be any bounded linear functional over $\mathfrak{B}_{c}$. Then $F$ restricted to $\mathfrak{B}_{r}$ is also linear and bounded. If $f=f(x) \in \mathfrak{B}_{r}$, let $F f=F_{1} f$ $+i F_{2} f$ be the decomposition of $F f$ into its real and imaginary parts. Then $F_{1} f$ and $F_{2} f$ are bounded linear functionals over $\mathfrak{B}_{r}$. Thus, if $F \in \mathfrak{B}_{c}{ }^{*}$ and $f \in \mathfrak{B}_{r}$, there exist $F_{1}, F_{2} \in \mathfrak{B}_{r}^{*}$ such that $F f=F_{1} f+i F_{2} f$. Now let $f \in \mathfrak{B}_{c}$, that is, $f=f_{1}+i f_{2}$, and let $F_{1}$ and $F_{2}$ be extended to $\mathfrak{B}_{c}$. Then it is easy to see that

$$
F\left(f_{1}+i f_{2}\right)=F_{1}\left(f_{1}+i f_{2}\right)+i F_{2}\left(f_{1}+i f_{2}\right) .
$$

In other words, $F=F_{1}+i F_{2}$ and each functional on $\mathfrak{B}_{c}$ is in the linear manifold of the extensions of the functionals on $\mathfrak{B}_{r}$.

Now consider an arbitrary bounded linear transformation $S$ defined over a real space $\mathfrak{B}_{r}{ }^{*}$. We extend $S$ to $\mathfrak{B}_{c}{ }^{*}$ by defining

$$
S\left(F_{1}+i F_{2}\right)=S F_{1}+i S F_{2} .
$$

Here $F_{1}$ and $F_{2}$ denote the extensions to $\mathfrak{B}_{c}$ of functionals on $\mathfrak{B}_{r}$. Note that by virtue of the preceding paragraph, every functional $F \in \mathfrak{B}_{c}{ }^{*}$ has the form $F=F_{1}+i F_{2}$. Also $S F_{1}$ and $S F_{2}$ denote the extension to $\mathfrak{B}_{c}$ of the functionals $S F_{1}$ and $S F_{2}$ defined originally over $\mathfrak{B}_{r}$. It may be seen that $S$ is now a bounded linear transformation over $\mathfrak{B}_{c}{ }^{*}$.

We apply this procedure to the projection $T$ defined in the preceding pages. By virtue of the linearity of this transformation, it is easy to show that $T$ is a projection over $\mathfrak{B}_{c}{ }^{*}: T^{2}=T$. At this point we extend the notion of an integral. We recall that a real integral $F$ over $\mathfrak{B}_{r}$ is a linear functional which can be expressed as a difference $F=F_{1}-F_{2}$ where the $F_{j}$ are positive and such that $f_{n} \uparrow f$ implies $F_{j} f_{n} \rightarrow F_{j} f, j=1,2$. A complex integral is a linear functional over $\mathfrak{B}_{c}{ }^{*}$ of the form $F=G+i H$ where $G$ and $H$ are real integrals. It is now clear that the range of $T$ is the manifold of complex integrals. The other property enunciated for $T$ in Theorem $\mathrm{A}$ is obviously valid. We have therefore

TheOREM B. In the space of linear functionals over the complex space $\mathfrak{B}_{c}$ there is a unique projection $T$ which has the properties:

(a) $T G=G$ if and only if $G$ is an integral.

(b) The transformations $T$ and $I-T$ are positive. 


\section{REFERENCES}

1. E. R. Lorch, Abstract 250t, Bull. Amer. Math. Soc., 60 (1954), 155.

2. M. A. Woodbury, Abstract 167t, Bull. Amer. Math. Soc., 56 (1950), 171.

3. K. Yosida and E. Hewitt, Finitely additive measures, Trans. Amer. Math. Soc., 72 (1952), $46-66$.

Barnard College

Columbia University

New York 